\title{
Antitumoral effect of IL-12 gene transfected via liposomes into B16F0 cells
}

\author{
Lucía Speroni $^{1}$, Julieta Gasparri ${ }^{1}$, Victoria de los A. Bustuoabad ${ }^{2}$, \\ Nadia S. Chiaramoni ${ }^{1}$, Andrzej Smagur ${ }^{3}$, Stanisław Szala ${ }^{3}$, María C. Taira ${ }^{1}$ and \\ Silvia del V. Alonso ${ }^{1 \bowtie}$ \\ ${ }^{1}$ Laboratorio de Biomembranas, Departamento de Ciencia y Tecnologia, Universidad Nacional de Quilmes, \\ Buenos Aires, Argentina; ${ }^{2}$ Instituto de la Visión; Hospital Oftalmológico Malvinas Argentinas; Buenos Aires, \\ Argentina; ${ }^{3}$ Department of Molecular Biology, Maria Skłodowska-Curie Memorial Cancer Center and Institute \\ of Oncology, Gliwice, Poland
}

Received: 19 December, 2008; revised: 15 April, 2009; accepted: 27 April, 2009 available on-line: 07 May, 2009

\begin{abstract}
Murine melanoma B16F0 cells were transfected with SA:DPPC:DOPE (2:1:1 molar ratio) liposomes associated with a plasmid encoding murine IL-12. Stearylamine, a cationic lipid, showed a greater transfection efficiency compared to DOTAP-containing liposomes. The lipid:DNA ratio was 2:1 $(w / w)$. Control groups were mock transfected or transfected with an empty plasmid (pNeo). pNeo or IL-12 transfected cells and controls were inoculated intradermically into the dorsal region of the foot or the lateral flank of C57BL6 mice. Results showed that IL-12 expression had a marked effect on in vivo growth of B16 melanoma tumors developed in both anatomic sites, significantly retarding their growth and prolonging host survival.
\end{abstract}

Key words: B16F0 cells, B16 melanoma, IL-12, liposomes, stearylamine

\section{INTRODUCTION}

Interleukin-12 (IL-12) plays an essential role in the interaction between the innate and adaptive arms of immunity. This cytokine is produced by phagocytic, dendritic, B, and possibly other accessory cells in response to infectious agents. It acts on $\mathrm{T}$ and NK cells by enhancing the generation and activity of cytotoxic lymphocytes and inducing the proliferation and production of other cytokines, especially IFN- $\gamma$. IL-12 is also the main cytokine responsible for the differentiation of $\mathrm{T}$ helper 1 cells, which are potent producers of IFN- $\gamma$ (Colombo et al., 2002). Particularly in B16 melanoma, IL-12 induces an antitumor effect dependent on $\mathrm{CD} 8^{+} \mathrm{T}$ cells (Brunda et al., 1993) and an antimetastatic effect dependent on NK cells (Kodama et al., 1999). Brunda et al. (1993) have clearly demonstrated that IL-12 exerts a strong anti-tumor activity based on the induction of IFN- $\gamma$ and the activation of effector cells such as $\mathrm{CD} 8^{+} \mathrm{T}$ and NK.

In this study B16F0 cells were transfected with a plasmid carrying the IL-12 gene associated with liposomes containing the cationic lipid stearylamine (SA). The efficiency of this cationic lipid in the transfection of eukaryotic cells and its low toxicity towards cells (Wang et al., 1996) makes it highly suitable for nucleic acid transfections. The relatively low cost of SA compared to other cationic lipids is another important consideration in routine applications. We evaluated the effect of IL-12 generated by $\mathrm{B} 16 \mathrm{~F} 0$ cells on the progression of melanoma tumors in C57BL6 mice. The progression of melanoma in C57BL6 mice was evaluated by inoculating transfected B16F0 cells into two anatomic sites: the lateral flank and the dorsal region of the foot.

${ }^{\square}$ Corresponding author: Silvia del V. Alonso, Laboratorio de Biomembranas, Departamento de Ciencia y Tecnologia, Universidad Nacional de Quilmes, Buenos Aires, Argentina; tel.: (5411) 43657100 ext. 323; fax: (5411) 4365 7132; e-mail: salonso@unq.edu.ar

Abbreviations: DOTAP, 1,2-dideoyl-3-trimethylammonium-propane; DPPC, 1,2-dipalmitoyl-glycero-3-phosphocholine; DOPE, 1,2-dioleoyl-glycero-3-phosphoethanolamine; MTT, methyl thiazol tetrazolium bromide; SA, stearylamine; SUVs, small unilamellar vesicles; $\mathrm{T}_{\text {regs }}$, regulatory $\mathrm{T}$ cells. 


\section{MATERIALS AND METHODS}

Liposome preparation. Lipids 1,2-dipalmitoyl-glycero-3-phosphocholine (DPPC), 1,2-dioleoyl-glycero-3-phosphoethanolamine (DOPE), and stearylamine (SA) were used in the liposome formulation SA:DPPC:DOPE (molar ratio 2:1:1). Lipids were dissolved in chloroform, and then the solvent was flash-evaporated (Bangham, 1972) and left under vacuum for $30 \mathrm{~min}$. Liposomes were constructed by adding phosphate buffer, $\mathrm{pH}$ 7.4. Small unilamellar vesicles (SUVs) were obtained by sonication with a titanium probe (Fisher Scientific) for 4 cycles of $30 \mathrm{~s}$ at $3 \mathrm{~W}$ with 30-s intervals, at room temperature under $\mathrm{N}_{2}$. Plasmid DNA association with SUVs was obtained by incubating DNA with SUVs for $1 \mathrm{~h}$ at room temp. The lipid:DNA ratio was 2:1 (w/w). Particle size was determined by light scattering using a 90Plus particle sizer (Brookhaven Instruments).

Plasmids. Plasmid pBCMGSNeo/mIL-12 contained sequences encoding both subunits of murine IL-12 (p35 and 540) separated by an IRES sequence, under the control of the CMV promoter. Plasmid pBCMGSNeo was used as control (Budryk et al., 2000).

Cell viability assay. Liposome cytotoxicity was tested in L929, Vero and B16F0 cell lines by the MTT assay (Wang et al., 2005). Briefly, $1.5 \times 10^{4}$ cells/well were seeded in a 96-well plate. After 24 $\mathrm{h}$, fresh culture media containing serial dilutions of liposomes were added. Cells incubated without liposomes were used as controls. After $5 \mathrm{~h}$ incubation, media were removed and replaced with $100 \mu \mathrm{l} /$ well of $0.5 \mathrm{mg} / \mathrm{ml}$ MTT solution. Plates were incubated for $2 \mathrm{~h}$ at $37^{\circ} \mathrm{C}$. Culture media were removed and $200 \mu \mathrm{l}$ of ethanol was added to dissolve MTT-formazan crystals developed in living cells. Cell viability was assessed by measuring absorbance at $570 \mathrm{~nm}$ on a MRX-TC Dynex Technologies micro titer plate reader.

In vitro transfection. $\mathrm{B} 16 \mathrm{~F} 0$ are murine melanoma cells which originate a weakly immunogenic tumor. The cells, a gift from the Laboratory of Molecular Oncology (Universidad Nacional de Quilmes) and obtained from ATCC (American Type Culture Collection, Rockville, MD), were grown in DMEM supplemented with $10 \%$ fetal bovine serum (FBS) until they were $60 \%$ confluent. Cultures were kept at $37^{\circ} \mathrm{C}$ in $5 \% \mathrm{CO}_{2}$ incubator. Culture media were replaced with DMEM without FBS, and lipid-DNA complexes were added. Liposomes (0.04 $\mu \mathrm{g} / \mathrm{ml}$ ) associated with pBCMGSNeo/mIL-12 (IL-12) or $\mathrm{pBCMGSNeo}(\mathrm{pNeo})$ were used for transfection. Control cells were mock transfected (control). An incubation period of $5 \mathrm{~h}$ was allowed for transfection to occur, and then culture media were replaced with DMEM plus 2\% FBS. Following an overnight period, cells were washed with PBS and trypsinized. To evaluate the efficiency of transfection, plasmid pCH110 encoding $\beta$-galactosidase reporter gene was used. Transfection efficiency was expressed as the percentage of blue stained cells (positive cells) over total cells after X-gal staining (Jacobsen et al., 2006).

Inoculation of transfected B16F0 cells. pNeo (or IL-12) transfected B16F0 cells $\left(2 \times 10^{5}\right)$ and controls were inoculated intradermically in the dorsal region of the foot or the lateral flank of C57BL6 mice (6-8 weeks old). The diameter of tumors growing in the lateral flank was measured with a caliper, and their volume was calculated according to the formula of Attia and Weiss (1966): $\mathrm{A}^{2} \times \mathrm{B} \times 0.4$, with $\mathrm{A}$ and $\mathrm{B}$ representing the smallest and the largest tumor diameters, respectively. Tumor progression in the dorsal region of the foot was followed using a spring-loaded caliper, and swelling was reported. Mouse survival was also recorded.

Statistical analysis. Data were assayed by ANOVA and Student's t-test. Differences between means were considered significant when yielding a $P<0.05$. Results are presented as means \pm S.D.

\section{RESULTS}

\section{Particle size determination}

As expected, liposome size was reduced with sonication. After DNA association with sonicated liposomes, the particle size in the major population did not change significantly (Table 1 ).

\section{Effect of liposomes on cell viability}

To test the effect of liposomes on cell viability, MTT assay was performed in different cell lines incubated with liposomes, with special interest in the effect on B16F0 cells. Vero and L929 cell lines were used as control non-tumor cells. Results of the cell viability assays after liposome incubation are expressed in Fig. 1. Liposome concentrations between $0.02-0.06 \mu \mathrm{g} / \mathrm{ml}$ reduced the viability by $10-30 \%$ in L929 and Vero cells, but did not affect the B16F0 cell line. Liposome concentrations higher than $0.13 \mu \mathrm{g} /$ $\mathrm{ml}$ reduced cell viability by $50 \%$ or less in all cell lines assayed. Based on these results, the range of

Table 1. Liposome particle size determination.

\begin{tabular}{lc}
\hline Sample & $\begin{array}{c}\text { Mean particle size }(\mathrm{nm}) \\
\text { of major population }(\%)\end{array}$ \\
\hline Non-sonicated liposomes & $3370(75 \%)$ \\
Sonicated liposomes & $433(60 \%)$ \\
Liposomes-plasmid DNA & $375(60 \%)$ \\
\hline
\end{tabular}




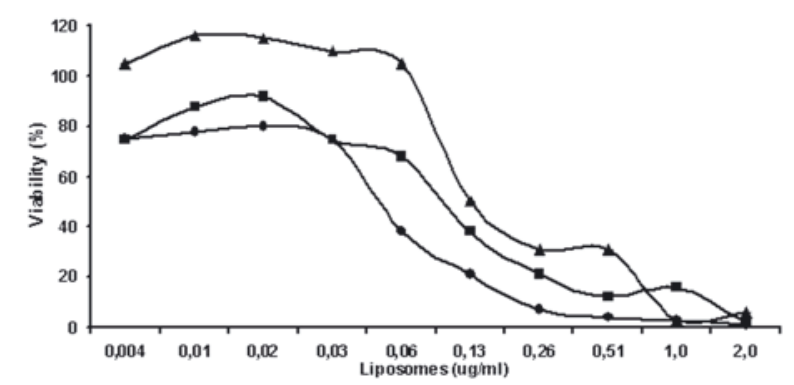

Figure 1. Cell viability assay.

Liposomes were incubated with L929 $(\bullet)$, Vero $(\boldsymbol{\square})$ and B16F0 ( $\mathbf{\Delta})$ cell lines and $5 \mathrm{~h}$ later MTT assay was performed.

concentrations between 0.02 and $0.06 \mu \mathrm{g} / \mathrm{ml}$ was selected for cell transfection assays.

In vitro transfection. The ability of liposomes to transfect B16F0 cells was evaluated using a plasmid encoding $\beta$-galactosidase reporter gene. In vitro transfection was assayed by X-gal staining. Transfection efficiency using SA:DPPC:DOPE liposomes was $13 \% \pm 0.62$. Liposomes containing DOTAP instead of SA as a cationic lipid showed a lower transfection efficiency for this cell line (not shown).

\section{Inoculation of transfected B16F0 cells}

The effect of IL-12 expression on tumor growth in two different anatomic sites was assayed by inoculating transfected B16F0 cells into the flank and the dorsal region of the foot of C57BL6 mice. Tumor progression is shown in Fig. 2. In the dorsal region of the foot (Fig. 2A), tumors originated from IL-12 transfected cells presented a slower progression compared to $\mathrm{pNeo}$ and control samples. On day 19, IL-12 tumors were smaller by 50\% than pNeo and controls. Similar results were obtained when B16F0 cells were intradermically injected into the lateral flank of mice (Fig. 2B). Tumor growth followed a similar pattern in all groups up to day 25 . From this day on, IL-12 tumors seemed to reach a plateau that lasted until day 30. Conversely, on day 30, pNeo and control tumors showed a peak growth while IL-12 tumors maintained a constant volume from day 33 until day 40. On day 40, tumor volume in pNeo and control samples was $40 \%$ larger than in IL-12 samples.

In the case of B16F0 cells injected intradermically into the dorsal region of the foot (Fig. 3A), it was observed that $\mathrm{pNeo}$ and control mice did not survive beyond day 18 . On the other hand, 100\% IL12 mice survived up to day 34, and 20\% IL-12 mice survived up to day 49. Groups of mice with B16F0 cells injected intradermically in the lateral flank are shown in Fig. 3B. On day 18, 80\% control and 50\%
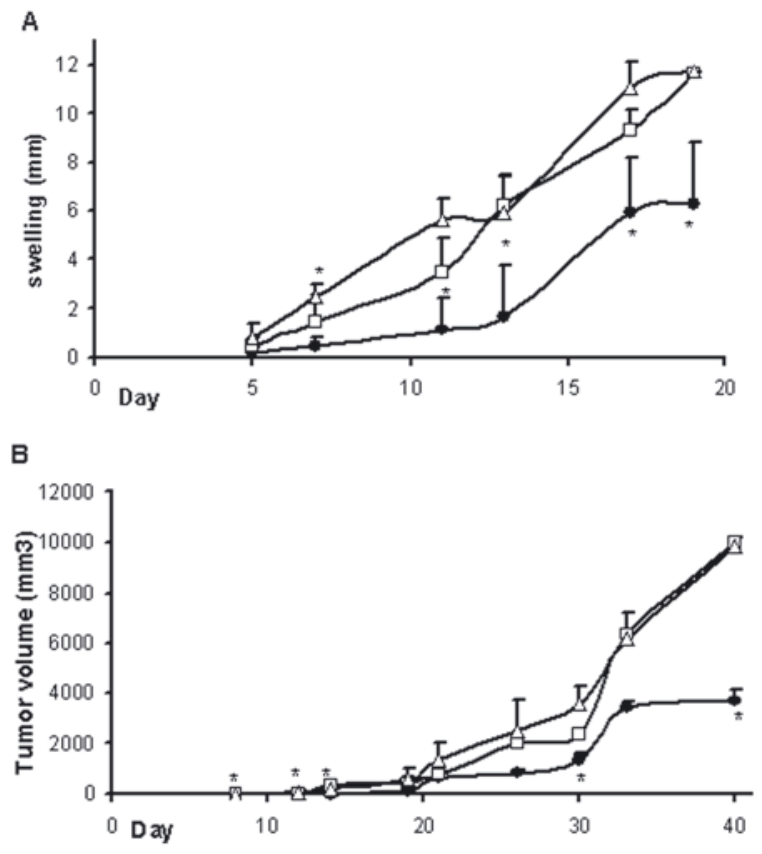

Figure 2. Tumor growth.

B16F0 cells transfected with plasmid encoding IL-12 $(\bullet)$, with empty plasmid $(\square)$ or mock transfected $(\Delta) \quad(n=5)$ were injected either intradermically into the dorsal region of the foot (A) or in the lateral flank (B). In both anatomic sites, tumors originated from IL-12-transfected cells presented slower progression compared to $\mathrm{pNeo}$ and control samples. Points, mean bars, \pm S.D. ${ }^{*} P<0.05$.
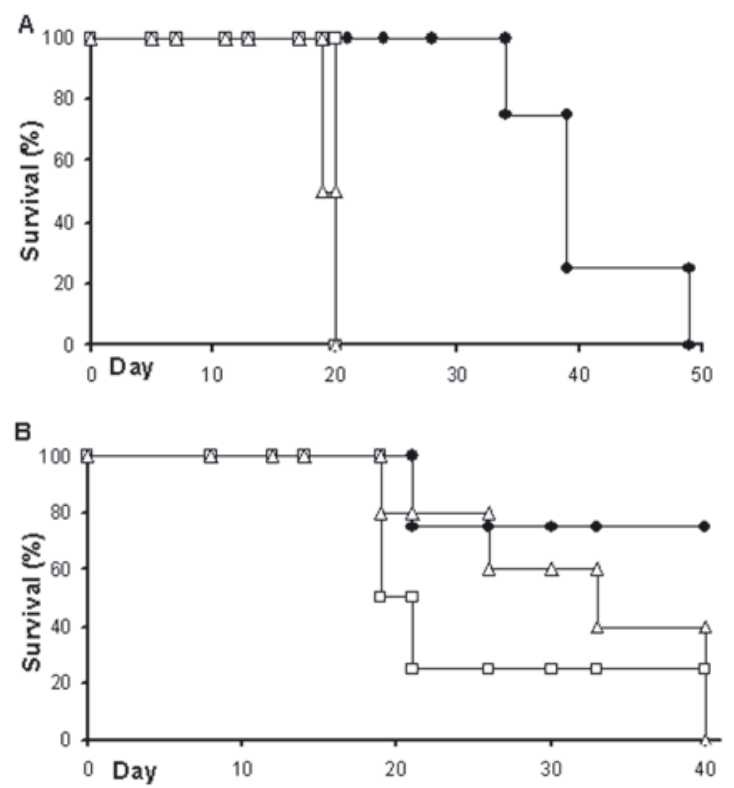

Figure 3. Mice survival.

C57BL6 mice were injected intradermically into the dorsal region of the foot (A) or into the lateral flank (B) with B16F0 cells transfected with plasmid encoding IL-12 $(\bullet)$, with empty plasmid $(\square)$ or mock transfected $(\Delta)(n=5)$. Prolonged host survival was observed in IL-12 group compared to controls. 
pNeo mice were alive, while $75 \%$ survival was observed in the IL-12 group until day 50 .

\section{DISCUSSION}

Liposomes-plasmid DNA complexes used for cell transfection were of comparable particle size to that used by Son et al. (2000), where complexes of 322-355 $\mathrm{nm}$ were efficient transfection vectors. Liposomes containing DC-6-14 (Asada et al., 2002) or Lipofectamin (Fu et al., 1998) have been used for transfection of B16 cells. In this work we chose SA as the cationic lipid because it showed a greater transfection efficiency compared to DOTAP-containing liposomes. A 13\% transfection efficiency of the B16F0 cell line with SA-containing liposomes was obtained. This value is not high compared to that obtained with viral transduction vectors. However, our results demonstrated that SA-containing liposomes could efficiently deliver plasmid DNA, which in turn showed a remarkable antitumor effect when the IL-12-encoding plasmid was transfected into B16F0 cells.

Results of the cell viability assay showed that B16F0 cells were not affected by liposomes in the concentration range used. This rules out the possibility that differences in tumor progression and survival between the experimental groups were due to tumor cell death during in vitro transfection.

This study shows that IL-12 expression has a marked effect on in vivo growth of B16 melanoma tumor originated from B16F0 cells transfected with the IL-12 gene. Both the tumors generated in the dorsal region of the foot and in the flank had a significantly retarded growth and in both cases the host survival was prolonged by IL-12 expression.

These results are in good agreement with previous reports showing that IL-12 had a strong anti-tumor effect on B16 melanoma and its variants. Lasek et al. (2004) developed genetically modified B78/IL-12 cells that, when injected, induced a strong activation of anti-tumoral mechanisms resulting in complete loss of tumorigenicity. Elzaouk et al. (2006) showed that mesenchymal stem cells (MSCs) stably transduced with a retroviral vector expressing IL-12 in a mouse melanoma model elicited a marked retardation in tumor growth and led to prolonged survival when injected into established subcutaneous melanoma in a therapeutic regimen.

IL-12 appeared to have a higher anti-tumoral activity than other cytokines assayed. Locally secreted IL-12 stimulates the proliferation and cytotoxicity of natural killer (NK) cells and T lymphocytes, and promotes the generation of Th1 effector cells. Due to chemokine induction, IL-12 also exerts antiangiogenic effects decreasing blood supply to tumors. The continuous local secretion of IL-12 at the tumor site prevents toxicity related to systemic administration of recombinant IL-12 (Switaj et al., 2004). However, other authors have demonstrated that both systemically delivered and locally produced IL-12 affects the cell types that mediate the anti-tumor immune response (Nanni et al., 1998; Smyth et al., 2000). Discrepant results obtained using metastatic B16F10 probably depend on the dose and timing of IL-12 injection. For instance, Switaj et al. (2004) showed that the administration of B78/IL-12 vaccine was not enough to inhibit tumor growth in spite of the generation of cytotoxic effector cells in the spleen.

Thus, the apparently discrepant results on the IL-12 effect on tumor growth could be explained by Prehn's (2007) hypothesis. According to this author, the immune reaction may either stimulate or inhibit tumor growth, depending on the local ratio between immune reactants and tumor cells. Prehn defines "immune reaction" as the algebraic sum of all the interacting parts involved in the complex response to an antigenic stimulus: antibody, $\mathrm{T}$ cells, $\mathrm{T}_{\text {regs' }} \mathrm{NK}$ cells, macrophages etc. In carcinogenesis, as in implanted tumors, stimulation or inhibition probably depend on the location of the immune response curve in the system. This location, in turn, would depend on the intrinsic immune capacity of the host and the immunogenicity of the tumor. Thus, the stimulation of the immune response should be greater than a certain threshold for tumor growth inhibition to occur (Prehn, 2007). In our view, the increase in local IL-12 expression above this threshold causes the immune response to shift towards tumor growth inhibition. Therefore, in agreement with Prehn's idea, to determine the position that relates tumor growth to the magnitude of the immune reaction on the nonlinear curve would be of importance for the development of therapeutic strategies.

\section{Acknowledgements}

We thank Dr. Oscar D. Bustuoabad for his valuable help and assistance. Financial support was provided by UNQ and CONICET.

\section{REFERENCES}

Asada H, Kishida T, Hirai H, Satoh E, Ohashi S, Takeuchi M, Kubo T, Kita M, Iwakura Y, Imanishi J, Mazda O (2002) Significant antitumor effects obtained by autologous tumor cell vaccine engineered to secrete interleukin 12 and IL-18 by means of the EBV/lipoplex. Mol Ther 5: 609-616.

Attia MA, Weiss DW (1966) Immunology of spontaneous mammary carcinomas in mice. V. Acquired tumor resistance and enhancement in strain-A mice infected with mammary tumor virus. Cancer Res 26: 1787-1800. 
Bangham AD (1972) Model membranes. Chem Phys Lipids 4: 386-392.

Brunda MJ, Luistro L, Warrier RR, Wright RB, Hubbard BR, Murphy M, Wolf SF, Gately MK (1993) Antitumor and antimetastatic activity of interleukin 12 against murine tumors. J Exp Med 178: 1223-1230.

Budryk M, Wilczyńska U, Szary J, Szala S (2000) Direct transfer of IL-12 gene into growing Renca tumors. Acta Biochim Polon 47: 385-391.

Colombo MP, Trinchieri G (2002) Interleukin-12 in antitumor immunity and immunotherapy. Cytokine Growth Factor Rev 13: 155-168.

Elzaouk L, Moelling K, Pavlovic J (2006) Anti-tumor activity of mesenchymal stem cells producing IL-12 in a mouse melanoma model. Exp Dermatol 15: 865-874.

Fu J, Zheng J, Fang W (1998) Interleukin-12 gene transfection into murine B16 melanoma cells suppresses tumorigenicity and decreases metastatic potential. Zhonghua Yi Xue Za Zhi 78: 627-629.

Jacobsen F, Mertens-Rill J, Beller J, Hirsch T, Daigeler A, Langer S, Lehnhardt M, Steinau HU, Steinstraesser L (2006) Nucleofection: a new method for cutaneous gene transfer? J Biomed Biotechnol Article ID 26060, 2006: 1-8.

Kodama T, Takeda K, Shimozato O, Hayakawa Y, Atsuta M, Kobayashi K, Ito M, Yagita H, Okumura K (1999) Perforin-dependent NK cell cytotoxicity is sufficient for anti-metastatic effect of IL-12. Eur J Immunol 29: 13901396.

Lasek W, Basak G, Switaj T, Jakubowska AB, Wysocki PJ, Mackiewicz A, Drela N, Jalili A, Kamiński R, Kozar K, Jakóbisiak M (2004) Complete tumour regressions induced by vaccination with IL-12 gene-transduced tu- mour cells in combination with IL-15 in a melanoma model in mice. Cancer Immunol Immunother 53: 363372.

Nanni P, Rossi I, De Giovanni C, Landuzzi L, Nicoletti G, Stoppacciaro A, Parenza M, Colombo MP, Lollini PL (1998) Interleukin 12 gene therapy of MHC-negative murine melanoma metastases. Cancer Res 58: 12251230.

Prehn R (2007) Immunostimulation and immunoinhibition of premalignant lesions. Theor Biol Med Model 4: 6 .

Son K, Patel D, Tkach D, Park A (2000) Cationic liposome and plasmid DNA complexes formed in serum-free medium under optimum transfection condition are negatively charged. Biochim Biophys Acta 1466: 11-15.

Smyth MJ, Taniguchi M, Street SE (2000) The anti-tumor activity of IL-12: mechanisms of innate immunity that are model and dose dependent. J Immunol 165: 26652670.

Switaj T, Jalili A, Jakubowska AB, Drela N, Stoksik M, Nowis D, Basak G, Golab J, Wysocki PJ, Mackiewicz A, Sasor A, Socha K, Jakóbisiak M, Lasek W (2004) CpG immunostimulatory oligodeoxynucleotide 1826 enhances antitumor effect of interleukin 12 gene-modified tumor vaccine in a melanoma model in mice. Clin Cancer Res 10: 4165-4175.

Wang D, Jing N, Lin Q (1996) Stearylamine liposome as a new efficient reagent for dna transfection of eukaryotic cells. Biochem Biophys Res Commun 226: 450-455.

Wang JC, Liu XY, Lü WL, Lee HS, Goh BC, Zhang Q (2005) The in vitro cytotoxicity and in vivo toxicity of doxorubicin antiresistant stealth liposomes. Yao Xue Хие Вао 40: 475-480. 